\title{
Copy-and-Paste in Medical Student Notes: Extent, Temporal Trends, and Relationship to Scholastic Performance
}

\author{
Ken Monahan ${ }^{1}$ Cheng Ye ${ }^{2}$ Edward Gould ${ }^{3}$ Meng $\mathrm{Xu}^{4}$ Shi Huang ${ }^{4}$ Anderson Spickard III ${ }^{2,5}$ \\ S. Trent Rosenbloom ${ }^{2,5,6}$ Joseph Coco $^{2}$ Daniel Fabbri ${ }^{2}$ Bonnie Miller ${ }^{7}$
}

${ }^{1}$ Division of Cardiovascular Medicine, Vanderbilt University Medical
Center, Nashville, Tennessee, United States
2 Department of Biomedical Informatics, Vanderbilt University
Medical Center, Nashville, Tennessee, United States
${ }^{3}$ Division of Nephrology, Vanderbilt University Medical Center,
Nashville, Tennessee, United States
${ }^{4}$ Department of Biostatistics, Vanderbilt University Medical Center,
Nashville, Tennessee, United States
${ }^{5}$ Division of General Internal Medicine, Vanderbilt University Medical
Center, Nashville, Tennessee, United States
6 Department of Pediatrics, Vanderbilt University Medical Center,
Nashville, Tennessee, United States
${ }^{7}$ Office of Health Sciences Education-School of Medicine, Vanderbilt
University Medical Center, Nashville, Tennessee, United States

Address for correspondence Ken Monahan, MD, Division of Cardiovascular Medicine, Vanderbilt University Medical Center, 1215 21st Avenue - Medical Center East 5th Floor, Nashville, TN 37232, United States (e-mail: ken.monahan@vumc.org).

Appl Clin Inform 2019;10:479-486.

\section{Abstract}

\section{Keywords \\ - Undergraduate Medical Education \\ - clinical clerkship \\ - documentation}

Background Medical students may observe and subsequently perpetuate redundancy in clinical documentation, but the degree of redundancy in student notes and whether there is an association with scholastic performance are unknown.

Objectives This study sought to quantify redundancy, defined generally as the proportion of similar text between two strings, in medical student notes and evaluate the relationship between note redundancy and objective indicators of student performance. Methods Notes generated by medical students rotating through their medicine clerkship during a single academic year at our institution were analyzed. A studentpatient interaction (SPI) was defined as a history and physical and at least two contiguous progress notes authored by the same student during a single patient's hospitalization. For some students, SPI pairs were available from early and late in the clerkship. Redundancy between analogous sections of consecutive notes was calculated on a 0 to $100 \%$ scale and was derived from edit distance, the number of changes needed to transform one text string into another. Indicators of student performance included United States Medical Licensing Exam (USMLE) scores.

Results Ninety-four single SPIs and 58 SPI pairs were analyzed. Redundancy in the assessment/plan section was high (40\%) and increased within individual SPIs (to 60\%; $p<0.001$ ) and between SPI pairs over the course of the clerkship (by $30-40 \%$; $p<0.001)$. Students in the lowest tertile of USMLE step II clinical knowledge scores had higher redundancy in the assessment/plan section than their classmates $(67 \pm 24 \%$ vs. $38 \pm 22 \% ; p=0.002)$.

\section{received}

March 21, 2019

accepted after revision

April 29, 2019 (c) 2019 Georg Thieme Verlag KG Stuttgart . New York
DOI https://doi.org/

10.1055/s-0039-1692402. ISSN 1869-0327. 
Conclusion During the medicine clerkship, the assessment/plan section of medical student notes became more redundant over a patient's hospital course and as students gained clinical experience. These trends may be indicative of deficiencies in clinical knowledge or reasoning, as evidenced by performance on some standardized evaluations.

\section{Background and Significance}

Redundancy in clinical notes, whether due to auto-importing of preexisting data or to "copy-and-paste" practices, has been recognized as a pitfall of electronic medical records (EMRs). ${ }^{1,2}$ The use of copy-and-paste is highly prevalent among physicians ${ }^{3}$ and many notes contain redundant text. ${ }^{4,5}$

During clinical rotations, medical students write notes frequently. Surveys suggest the use of auto-importing and copy-and-paste by medical students is essentially ubiquitous and the vast majority of students have observed supervising residents and attending physicians engaging in such practices. ${ }^{6}$ This behavior perpetuates redundant documentation and reduces the potential for student notes to serve as educational tools, which has been a recognized goal of medical records for at least half a century. ${ }^{7}$ Although not studied specifically in students, copy-and-paste practices have also been associated with diagnostic errors and suboptimal treatment outcomes. ${ }^{8,9}$

Formal instruction on note writing during medical school may assist in breaking this cycle. However, prior to embarking on such a significant curricular initiative, quantifying the extent of redundancy in medical student notes and any impact on medical school performance is needed to provide justification and to identify potential targets for intervention. Therefore, using an established indicator of redundancy, we analyzed a cohort of notes generated by students rotating through the inpatient portion of their medicine clerkship during a single academic year at our institution.

\section{Objectives}

Based on prior work that focused on resident documentation, ${ }^{10}$ we sought to assess whether student notes become more redundant over the course of a given admission. Redundancy in notes, particularly in the assessment/plan section, may indicate a relative lack of emphasis by students on solidifying via documentation their understanding of the clinical course of patients they are following, which could be reflected in measures of student performance. As such, we aimed to evaluate the hypothesis that students with more redundant notes would have lower performance than those with less redundant notes.

\section{Methods}

The study was approved by the Vanderbilt Medical Center Institutional Review Board.

\section{Cohort of Student-Patient Interactions}

On the medicine clerkship, students document their encounters with patients in the form of an admission history and physical $(\mathrm{H}+\mathrm{P})$ and daily progress notes written via free text into the formal EMR. These notes are automatically copied directly from the EMR into a database maintained by the Department of Bioinformatics for instructional purposes. $^{11}$ From this database, all inpatient notes written during the 2012 to 2013 academic year were examined; this was the most recent year for which a complete set of notes was available.

A student-patient interaction (SPI) was defined as a series of notes written by the same student on a given patient during a single admission and consisted of: (1) an admission $\mathrm{H}+\mathrm{P}$; and (2) at least two consecutive daily progress notes contiguous with the $\mathrm{H}+\mathrm{P}$ (i.e., $\mathrm{H}+\mathrm{P}$ written on hospital day \#1 with the progress notes written on days \#2 and 3). Therefore, an SPI contained at least three notes. SPIs involving interservice transfers or intensive care unit patients were excluded due to the existence of a considerable number of notes prior to the creation of the student $\mathrm{H}+\mathrm{P}$.

If a student had more than one eligible SPI, one was selected at random for the main analysis. If a student had two eligible SPIs with one occurring during the first one-third of the clerkship and the second occurring during the last-third, those SPI pairs formed an additional cohort to assess changes in redundancy during the clerkship. For approximately threequarters of these pairs, one SPI occurred while the student was rotating on a general medicine team and the other occurred during a subspecialty rotation. The remainder of the pairs consisted of two subspecialty SPIs. The proportion of clerkship completed at the time of an SPI was calculated as the interval between the day the clerkship began and the day the $\mathrm{H}+\mathrm{P}$ was written divided by the duration of the clerkship (12 weeks). A rotation number (1-4) was designated based on when in the academic year each student was assigned to the medicine rotation (i.e., 1 is the earliest 12 -week block and 4 is the latest).

\section{Note Components and Comparisons}

Comparisons of note characteristics were performed between discrete subsections, rather than between entire notes as reported previously. ${ }^{10}$ For the $\mathrm{H}+\mathrm{P}$, medical history/problem list, medications, allergies, family history, and social history were included. The "initial progress note (IPN)" was defined as the first progress note following the $\mathrm{H}+\mathrm{P}$ (i.e., the note for hospital day \#2). The "next progress note (NPN)" was defined as the note following the IPN (i.e., the note for hospital day \#3). 
Table 1 Note types, sections, and comparisons

\begin{tabular}{|l|l|l|}
\hline $\begin{array}{l}\text { Current } \\
\text { document }\end{array}$ & $\begin{array}{l}\text { Prior } \\
\text { document }\end{array}$ & $\begin{array}{l}\text { Sections for } \\
\text { comparison }\end{array}$ \\
\hline $\begin{array}{l}\text { History and } \\
\text { physical exam }\end{array}$ & $\begin{array}{l}\text { Patient } \\
\text { summary }\end{array}$ & $\begin{array}{l}\text { Medical history/ } \\
\text { Problem list }\end{array}$ \\
\hline & & Medications \\
\hline & & Allergies \\
\hline & & Family history \\
\hline $\begin{array}{l}\text { Initial progress } \\
\text { note }\end{array}$ & $\begin{array}{l}\text { History and } \\
\text { physical exam }\end{array}$ & Social history \\
\hline & & Assessment/Plan \\
\hline $\begin{array}{l}\text { Next progress } \\
\text { note }\end{array}$ & $\begin{array}{l}\text { Initial progress } \\
\text { note }\end{array}$ & Physical examination \\
\hline & & Assessment/Plan \\
\hline
\end{tabular}

The key progress note sections were physical examination and assessment/plan. For each SPI, a patient summary was also used in the analysis. Note templates used by medical students auto-import data from the patient summary, which contains components of the history that can be updated by anyone involved in the patient's care. When a change is made to the patient summary, the new version is saved. The patient summary most proximal to the hospitalization of interest was used and individual subsections from this patient summary were compared to the analogous subsections of the $\mathrm{H}+\mathrm{P}$. The specific note section pair comparisons are displayed in -Table 1.

If an SPI contained more than two progress notes, then the $n$th and $n-1$ th notes were compared in similar fashion as the "NPN versus IPN." Vital signs were omitted from the analysis as these are inherently variable from day to day and thus may systematically bias assessments of redundancy.

\section{Identification and Selection of Note Sections}

For a given note within each SPI, the sections of interest were identified by manual review. Five reviewers, either 4th year medical students or senior-level nurse practitioner students, were each assigned randomly to an equal portion of the SPIs. These reviewers worked independently of one other and did not collaborate. Each note was loaded into a modified version of the software platform PYBOSSA, an open-source tool used to manage crowdsourcing efforts, within which custom software was used to mark the beginning and end of a given note section. ${ }^{12}$ The associated text was tagged and saved with the appropriate label, which incorporated the note section, document type, and a unique identifier for the SPI. To facilitate evaluation of interobserver reliability, each reviewer's task list was constructed such that there was a degree of overlap with another reviewer.

\section{Redundancy Metrics, Student Characteristics, and Performance Measures}

The main redundancy metric was based on the Levenshtein edit distance, a parameter that tabulates the number of additions, deletions, and substitutions needed to transform one text string into another. ${ }^{13}$ As changes to notes typically include additions or deletions of entire words rather than individual characters in the same words, we used word-level edit distance for this analysis. This edit distance can be normalized by the size (number of words) of the longer string such that it takes on a value between 0 and 1 , where a normalized edit distance of 0 denotes that two strings are identical and a value of 1 indicates that they are entirely unique. For two analogous note sections, we defined redundancy as 1 -normalized word-level edit distance-such that this term (multiplied by 100) represents the percentage of content that the 2 sections have in common. This measure quantifies redundancy of a note section pair from the same SPI (i.e., the physical examination of the IPN and the physical examination of the $\mathrm{H}+\mathrm{P}$ ).

Student characteristics were obtained from the Office of Undergraduate Medical Education (UME) and included date of birth, gender, and undergraduate major. Majors were grouped into engineering or physical/biological science or nonengineering/science.

Objective measures of student performance were obtained from the Office of UME and the Registrar's Office. These included Alpha Omega Alpha (AOA) status, medicine clerkship grades (honors, high pass, pass, fail), medicine shelf exam scores, and United States Medical Licensing Exam (USMLE) Step I and II clinical knowledge (CK) scores. Using national USMLE data, raw scores were converted into percentiles. All SPIs analyzed in this study occurred after the students took USMLE Step I, but prior to them taking USMLE step II CK.

\section{Statistical Analysis}

Comparisons between two continuous variables were made with the Mann-Whitney test and comparisons between more than two continuous variables were made with the Kruskal-Wallis test as the pertinent covariates were not normally distributed. Comparisons of redundancy between note section pairs were modeled as paired occurrences and thus the Wilcoxon matched-pairs signed rank test was used. The Pearson's correlation coefficient was used to assess the relationship between two continuous variables. These analyses were completed in GraphPad Prism 7.02 (GraphPad Inc; La Jolla, California, United States); $p$-value of $<0.05$ was considered statistically significant.

Redundancy was used to evaluate interobserver reliability of note section identification. As the reviewers were given identical training and instructions and worked independently, all comparisons assessed by two reviewers were treated as if they were completed by the same two reviewers. If the content of a given pair of note sections was selected by two reviewers in the same manner, the two resultant redundancy values should be identical. Therefore, for the subset of note section pairs that was assessed by two reviewers, the redundancy values from the two reviews were used to calculate the intraclass correlation coefficient (R Project version 3.5.1; R Foundation; Vienna, Austria). 
Table 2 Student characteristics and medical school performance

\begin{tabular}{|l|l|}
\hline Characteristic & Main cohort $(\boldsymbol{n}=\mathbf{9 4})$ \\
\hline Age at initial SPI (y) & $26 \pm 2$ \\
\hline Female (\%) & 53 \\
\hline Undergraduate major (\%) & \\
\hline Science/engineering & 76 \\
\hline Nonscience/engineering & 24 \\
\hline AOA society (\%) & 17 \\
\hline Medicine clerkship grade (\%) & \\
\hline Honors & 33 \\
\hline High pass & 64 \\
\hline Medicine shelf exam score & $81 \pm 8$ \\
\hline USMLE Step I & \\
\hline Score & $238 \pm 17$ \\
\hline Percentile & $68 \pm 22$ \\
\hline USMLE Step II CK & \\
\hline Score & $247 \pm 15$ \\
\hline Percentile & $63 \pm 25$ \\
\hline
\end{tabular}

Abbreviations: AOA, Alpha Omega Alpha; CK, clinical knowledge; SPI, student-patient interaction; USMLE, United States Medical Licensing Exam. Note: Continuous covariates are mean \pm standard deviation.

\section{Results}

\section{Cohort Characteristics}

Ninety-four students contributed a single SPI and 58 of those students contributed two SPIs. -Table 2 displays basic demographics, undergraduate major, and medical school performance metrics. Students were typically in their mid20s and slightly more than half were female. Approximately three-quarters of students majored in engineering or a physical/biologic science. Less than $20 \%$ of students were members of AOA and nearly all students earned honors or high pass in the medicine clerkship. Scores for UMSLE Step I and II CK were in the mid-upper 60th percentile.

\section{Interobserver Variability}

A total of 135 pairs of redundancy values were used to calculate the intraclass correlation coefficient. These included contributions from all the note section pair combinations described above. The overall intraclass correlation coefficient was 0.80 and the $95 \%$ confidence intervals were 0.73 to 0.86 . This level of agreement is comparable to recent studies of note redundancy. ${ }^{14,15}$

\section{Redundancy between the Admission H + P and Patient Summary}

The redundancy of comparable sections of the admission $\mathrm{H}+\mathrm{P}$ and patient summary is shown in - Fig. 1. Most sections were approximately $50 \%$ redundant with the allergies section exceeding $60 \%$ redundancy. Sixteen SPIs did not have a preexisting patient summary as the admission used for the SPI was the patient's first encounter with our medical center. Of

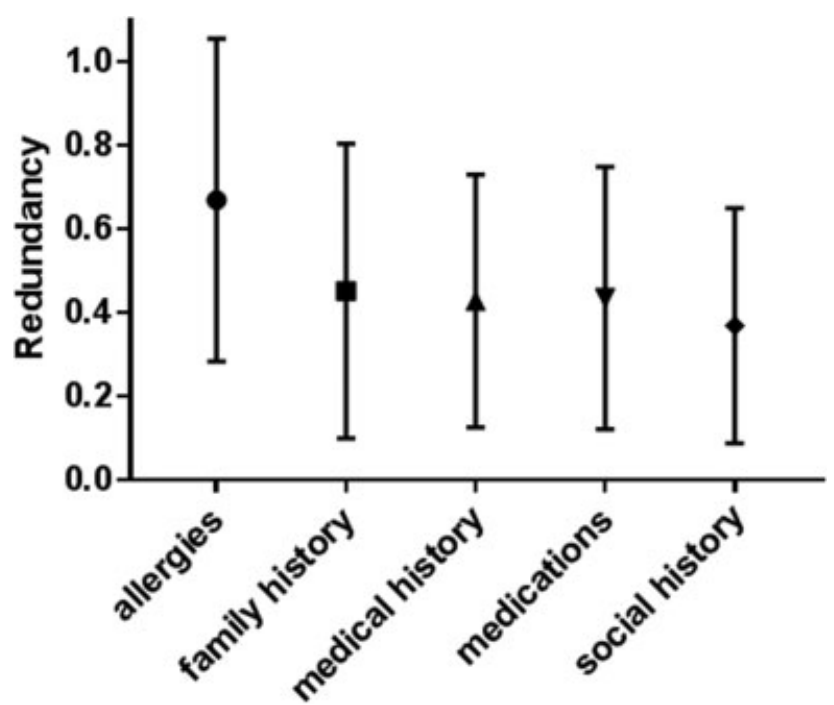

Fig. 1 Redundancy between the admission history and physical and patient summary. Most sections show redundancy of approximately $50 \%$ with the allergy section exceeding $60 \%$. Sixteen student-patient interactions (SPIs) did not have a patient summary as the admission used for the SPI was the patient's first encounter with our medical center. Data are displayed as mean \pm standard deviation.

note, approximately $50 \%$ of the allergy section comparisons yielded completely redundant results. Nearly $60 \%$ of family history comparisons and more than $30 \%$ of medical history comparisons had greater than $80 \%$ redundancy.

\section{Redundancy over the Course of an SPI}

The temporal trends of redundancy during an SPI for the physical examination (panel A) and assessment/plan (panel B) sections are displayed in - Fig. 2. For both sections, the redundancy between the IPN and the $\mathrm{H}+\mathrm{P}$ is approximately 35 to $40 \%$. Redundancy between the NPN and the IPN is significantly higher $(p<0.001)$ for both sections (nearly $80 \%$ for physical exam and nearly $60 \%$ for assessment/plan). In the subset of SPIs $(n=43)$ that had a subsequent progress note (i.e., from hospital day \#4), redundancy between this note and the prior note was nearly identical to that between the next and IPNs. When stratified by rotation number (1: $n=25 ; 2: n=23 ; 3: n=26 ; 4: n=18)$, the increase in assessment/plan redundancy within an SPI remained highly significant during all blocks. In addition, there was no difference in assessment/plan redundancy based on rotation number for either note pair (i.e., IPN vs. H + P or NPN vs. IPN).

\section{Analysis of Paired SPIs}

The "early" SPI in a given pair occurred after $26 \pm 10 \%$ of the clerkship had elapsed and the "late" SPI occurred $74 \pm 7 \%$ into the rotation. For the early and late SPIs, - Fig. 3 shows redundancy for the assessment/plan section of the IPN/H + $\mathrm{P}$ and the NPN/IPN. Consistent with the main cohort results, redundancy increases significantly over time for both the early and late SPIs $(p<0.001)$. Additionally, at both time points evaluated, redundancy is significantly higher for the late SPI comparisons relative to the early SPI comparisons (by $\sim 30-40 \%$; $p<0.001$ for both). 

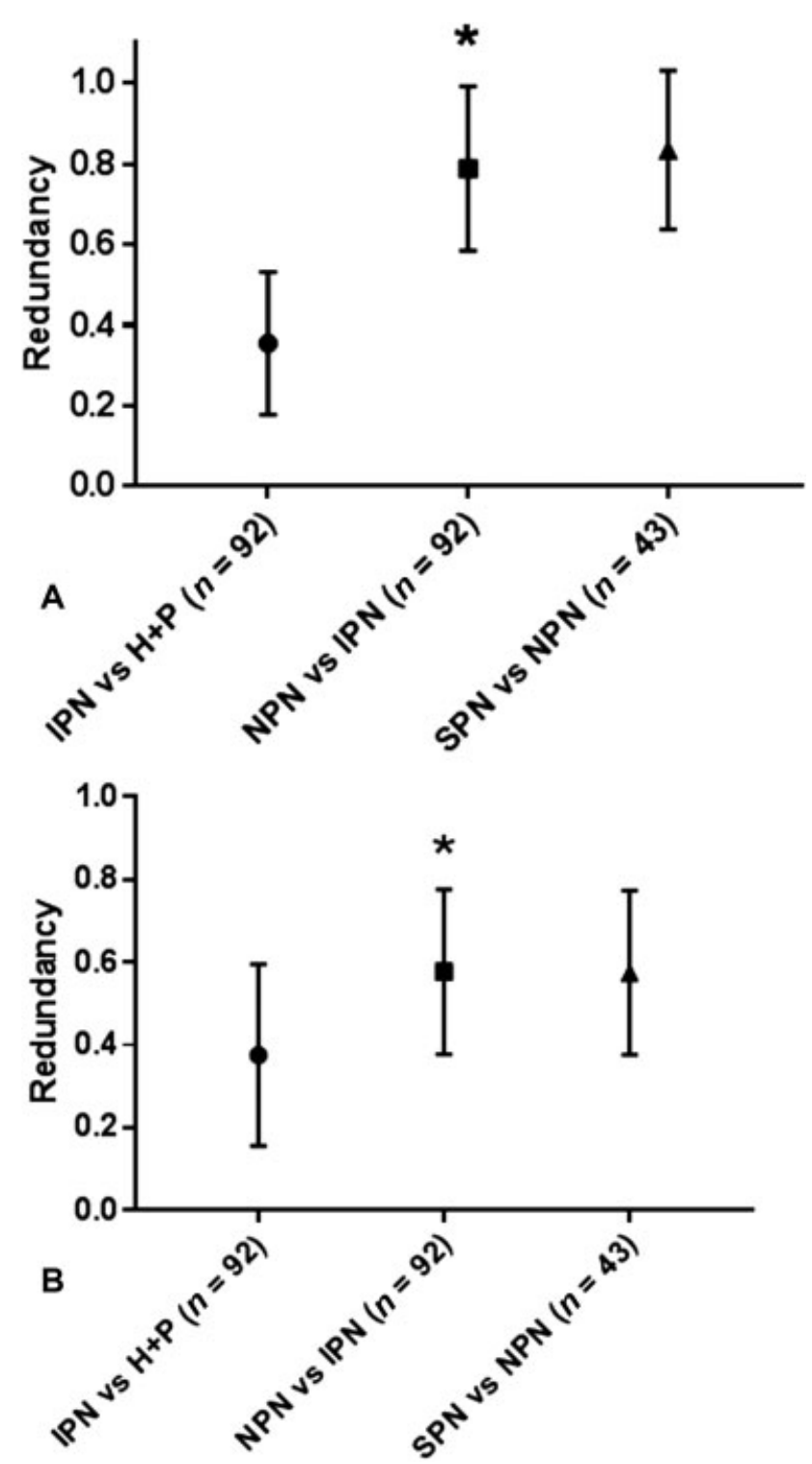

Fig. 2 Redundancy over the course of a student-patient interaction. For both the physical examination (A) and the assessment/plan (B), redundancy increases significantly between the IPN/H + P and the NPN/IPN comparisons. For the subset of student-patient interaction with an SPN $(n=43)$, the redundancy between the SPN and NPN is no different than the redundancy between the NPN and IPN. Data are displayed as mean \pm standard deviation. $\mathrm{H}+\mathrm{P}$, history and physical examination; IPN, initial progress note; NPN, next progress note; SPN, subsequent progress note.

\section{Redundancy and Measures of Medical Student Performance}

There was no significant difference between redundancy in the assessment/plan section when stratified by AOA status, undergraduate major, or clerkship grade. Similarly, the correlation between redundancy and shelf exam score was not significant.

-Fig. 4 illustrates the relationship between redundancy of the assessment/plan section of the IPN/H + P comparison and USMLE Step II CK percentile. For the upper two-thirds of scores, redundancy is essentially uniformly distributed. However, within the lowest tertile of scores, there is a cluster

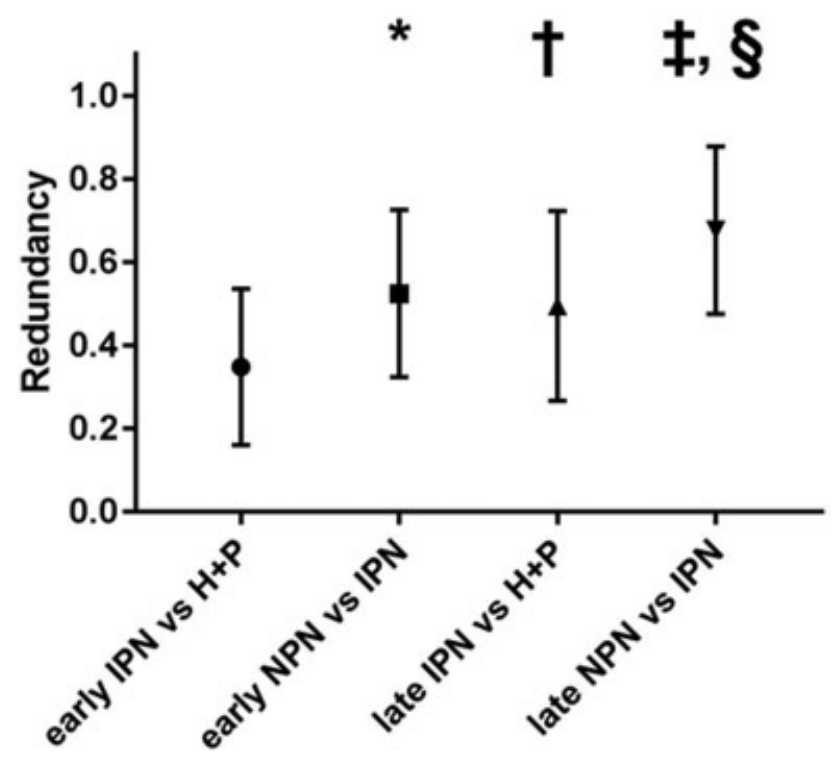

Fig. 3 Redundancy in student-patient interactions over the course of the medicine clerkship. For the assessment/plan section, redundancy increases not only over the course of a given student-patient interaction, but over the course of the clerkship as well. Data are taken from 58 pairs of studentpatient interactions that occurred after $26 \pm 10 \%$ (early) and $74 \pm 7 \%$ (late) of the clerkship had elapsed. Data are displayed as mean \pm standard deviation. $\mathrm{H}+\mathrm{P}$, history and physical examination; IPN, initial progress note; NPN, next progress note; SPN, subsequent progress note. ${ }^{*} p<0.001$ compared to early IPN vs. H + P. $^{\dagger} p<0.001$ compared to early IPN vs. $\mathrm{H}+\mathrm{P} .{ }^{\ddagger} p<0.001$ compared to late IPN vs. H + P. ${ }^{\S} p<0.001$ compared to early NPN vs. IPN.

of higher redundancy (panel A) and redundancy is significantly higher in the lowest tertile than in the upper tertiles $(67 \pm 24 \%$ vs. $38 \pm 22 \% ; p=0.002$ ) (panel B). Similar results were found for the assessment/plan section of the NPN/IPN comparison. Comparable findings were not observed with USMLE Step I.

\section{Discussion}

This study quantifies the redundancy present in student notes over the course of patients' time in the hospital and at different time points during the medicine clerkship. Exploratory analyses suggest a potential association between redundancy and medical school performance.

While some redundancy in the $\mathrm{H}+\mathrm{P} /$ patient summary comparison can be anticipated, ${ }^{6,16}$ two sections suggest a degree of pervasiveness that may be counterproductive. In nearly $50 \%$ of SPIs, the allergy section was entirely redundant, meaning that no changes were made to the auto-imported text. Upon further review, most of these sections contained information other than some variant of "no known drug allergies," suggesting that this information may not have been verified by the student, a phenomenon previously reported in a cohort of dermatology residents. ${ }^{17}$ More than $30 \%$ of the medical history/problem list sections had at least $80 \%$ redundancy, meaning that a substantive portion of medical students are not actively constructing patients' medical histories first-hand, an important skill to 

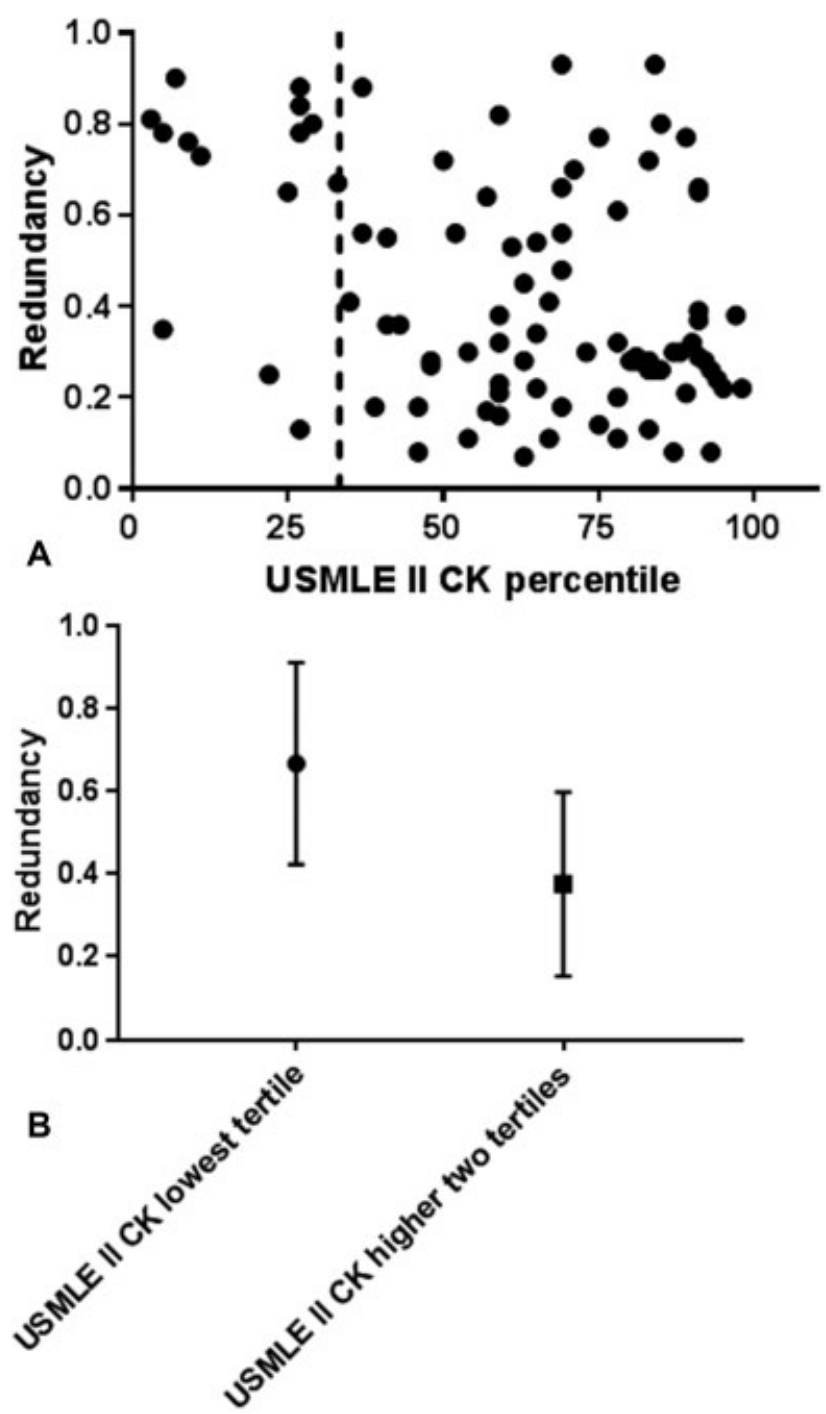

Fig. 4 Redundancy and USMLE Step II clinical knowledge scores. (A) There is a cluster of high-redundancy within the lowest tertile of USMLE Step II clinical knowledge scores. The lowest tertile is to the left of the dashed vertical line. (B) Redundancy within the lowest tertile is significantly higher than within the upper two tertiles $(p=0.002)$. Redundancy is for the assessment/plan section of the initial progress note/history and physical comparison. Data in (B) are displayed as mean \pm standard deviation. CK, clinical knowledge; USMLE, United States Medical Licensing Examination.

learn when trying to formulate differential diagnoses for the present illness.

Increasing redundancy in the assessment/plan section as the hospital course progresses could reflect the truly static nature of some SPIs. However, the comparisons examined here are relatively early in the hospital stay when patient evaluations and status can be at their most dynamic. For a given SPI, higher redundancy over time may reflect the inherently greater amount of material upon which to draw for the next note, similar to findings from analyses of house staff documentation..$^{10}$ Likewise, accumulated observations by students of documentation habits among house staff and attending physicians may contribute to the increased redundancy seen later in the clerkship relative to earlier. Increasing redundancy over the course of an SPI was independent of exposure to prior rotations, suggesting that habits learned on other services and "note fatigue" are not primarily responsible for this finding. Students may be learning "efficiency" at the expense of honing clinical reasoning through serial exposition of their patients' course. At the student level, such efficiency is less of a priority as students typically follow a relatively small subset of their teams' patients and only certain sections of student notes may be used for billing and thus they are largely exempt from any billing-related requirements imposed upon their content.

The findings regarding USMLE scores suggest that notebased metrics could compliment traditional tools for identifying subgroups of students who may be struggling with CK and its application. A similar relationship was not shown between note redundancy and USMLE Step I, an exam geared more toward testing basic science principles, suggesting that note analytics may be more reflective of clinical, rather than fact-based, knowledge. Likewise, there was no association between note redundancy and AOA status, clerkship grade, or shelf exam score. Subjective criteria are incorporated into selection for AOA and these attributes may not be captured by note characteristics. Student evaluations contain subjective elements, which may confound the relationship between clerkship grades and note redundancy as well. The discordance between medicine shelf exam and USMLE Step II CK scores with regards to their association with note redundancy is more challenging to reconcile, as both are objective continuous measures of CK. A potential explanation may lie in how each exam is perceived to impact applications for residency. The score on the shelf exam contributes to the clerkship grade, which in turn, influences the strength of an application for residency, particularly if a student is applying in internal medicine. In our cohort, the USMLE Step II CK exam was taken in the final year of medical school and the actual score may not have been a part of, or factored substantively into, the residency application. Most students took this exam either after residency interviews had concluded or proximal enough to interviews such that scores were not available during the interview process, plausibly leading to the perception that only the binary result (pass/fail) was ultimately important. Thus, if students are more incentivized to prepare for the shelf exam, the results may be out of proportion to the effort put forth in their notes, particularly for lower-performing individuals. Similar logic can be applied to USMLE Step I, which was taken by all students prior to beginning the 3rd year. Less preparation for the "lower-stakes" USMLE Step II CK exam may permit note characteristics to provide a more direct window into performance than is otherwise possible.

This study has several limitations. The main analysis was limited to a single SPI during a single clerkship in a single academic year at a single institution. This SPI may not be representative of a given student's note-writing tendencies, although the paired SPI analysis mitigates this shortcoming to some extent. The analysis emphasized the assessment/ plan section for two main reasons: (1) it is the first section 
of a note to be read and the section that providers spend the most time reading ${ }^{18}$; and (2) it was thought that this part of the note would be most reflective of idea synthesis and integration. Students may have displayed these cognitive attributes elsewhere in their notes, which would not have been reflected in our results. Similarly, we did not analyze auto-imported lab and test results and whether these were interpreted and integrated into the assessment/plan; such analysis could also provide insight into students' thought processes. Furthermore, it is also possible that subtle changes in the recorded assessment/plan, which would result in high redundancy, may have represented the result of considerable synthetic and integrative thought/discussion. Redundancy is a very basic metric and does not comment upon the conceptual complexity of note content. Other metrics may more accurately capture such higherlevel attributes. The key measure of student performance used in this study, USMLE Step II CK score, is not a perfect indicator of CK and reasoning, but it is available for all students and is standardized, thus increasing the applicability to medical students across the country. Our institution's EMR provides a "reuse" option, wherein the prior day's note can be cloned to form a template for the current day's note. The usage rate of this tool in our cohort is unknown, which limits the ability to estimate the degree to which redundancy may have been enabled by the design of the EMR itself. However, even if redundancy is entirely a surrogate for the "reuse" of prior notes, the range of redundancy within the cohort suggests the response by students to the availability of this feature is not uniform and thus the propensity to use it may provide insight that compliments traditional methods of student evaluation.

\section{Conclusion}

This study demonstrates that, within the medicine clerkship, medical student notes contain a high level of redundancy that increases over the course of a student's interaction with a given patient and over the course of the clerkship for a given student. Furthermore, high redundancy may indicate a relative deficiency in $\mathrm{CK}$ and/or reasoning, at least as measured by some standardized testing. To further evaluate and potentially address these observations, design and implementation of documentation-based initiatives in UME seems reasonable.

\section{Clinical Relevance Statement}

This demonstration of high redundancy in medical student notes and the association of note redundancy with lower scores on standardized tests of clinical knowledge/reasoning may motivate modifications to medical school curricula that focus on dedicated instruction in note writing. If this approach is implemented and found to be beneficial, the clinical care provided by future physicians may be enhanced by improvements in clinical reasoning and communication via more thoughtful and less redundant documentation.

\section{Multiple Choice Questions}

1. When comparing information from the Patient Summary and the History and Physical Examination, which section shows the greatest degree of redundancy?

a. Problem list.

b. Allergies.

c. Family history.

d. Social history.

Correct Answer: The correct answer is option b. The allergies section was $>60 \%$ redundant with the other sections having approximately 50\% redundancy.

2. With which indicator of student performance did note redundancy show a statistically significant relationship?

a. Medicine shelf exam.

b. Alpha Omega Alpha status.

c. USMLE Step I.

d. USMLE Step II CK.

Correct Answer: The correct answer is option d. Note redundancy was higher for those students that scored in the bottom one-third on the USMLE Step II CK exam compared to those who scored in the upper two-thirds. There were no statistically significant relationships between note redundancy and the other indicators of student performance.

Funding

None.

Conflict of Interest

None declared.

\section{Acknowledgments}

The authors thank Toufeeq Ahmed, PhD, for assistance with assembling the database of medical student notes, the Office of the University Registrar, as well as Regina Russell and colleagues in the Office of Undergraduate Medical Education for providing medical student information, and Jesse Wrenn, MD, PhD, for thoughtfully reviewing an earlier version of the manuscript.

\section{References}

1 Hirschtick RE. A piece of my mind. Copy-and-paste. JAMA 2006; 295(20):2335-2336

2 Siegler EL, Adelman R. Copy and paste: a remediable hazard of electronic health records. Am J Med 2009;122(06):495-496

3 Bowman S. Impact of electronic health record systems on information integrity: quality and safety implications. Perspect Health Inf Manag 2013;10:1c

4 Weir CR, Hurdle JF, Felgar MA, Hoffman JM, Roth B, Nebeker JR. Direct text entry in electronic progress notes. An evaluation of input errors. Methods Inf Med 2003;42(01):61-67

5 Hammond KW, Helbig ST, Benson CC, Brathwaite-Sketoe BM. Are electronic medical records trustworthy? Observations on copying, pasting and duplication. AMIA Annu Symp Proc 2003;2003:269-273

6 Heiman HL, Rasminsky S, Bierman JA, et al. Medical students' observations, practices, and attitudes regarding electronic health record documentation. Teach Learn Med 2014;26(01):49-55

7 Weed LL. Medical records that guide and teach. N Engl J Med 1968; 278(11):593-600 
8 Singh H, Giardina TD, Meyer AN, Forjuoh SN, Reis MD, Thomas EJ. Types and origins of diagnostic errors in primary care settings. JAMA Intern Med 2013;173(06):418-425

9 Turchin A, Goldberg SI, Breydo E, Shubina M, Einbinder JS. Copy/ paste documentation of lifestyle counseling and glycemic control in patients with diabetes: true to form? Arch Intern Med 2011; 171(15):1393-1394

10 Wrenn JO, Stein DM, Bakken S, Stetson PD. Quantifying clinical narrative redundancy in an electronic health record. J Am Med Inform Assoc 2010;17(01):49-53

11 Spickard A III, Ridinger H, Wrenn J, et al. Automatic scoring of medical students' clinical notes to monitor learning in the workplace. Med Teach 2014;36(01):68-72

12 Ye C, Coco J, Epishova A, et al. A crowdsourcing framework for medical data sets. AMIA Jt Summits Transl Sci Proc 2018; 2017:273-280

13 Levenshtein VI. Binary codes capable of correcting deletions, insertions, and reversal. Sov Phys Dokl 1966;10:707-710
14 Zhang R, Pakhomov SV, Lee JT, Melton GB. Using language models to identify relevant new information in inpatient clinical notes. AMIA Annu Symp Proc 2014;2014:1268-1276

15 Zhang R, Pakhomov SVS, Arsoniadis EG, Lee JT, Wang Y, Melton GB. Detecting clinically relevant new information in clinical notes across specialties and settings. BMC Med Inform Decis Mak 2017; 17(Suppl 2):68

16 Rizvi RF, Harder KA, Hultman GM, et al. A comparative observational study of inpatient clinical note-entry and reading/retrieval styles adopted by physicians. Int J Med Inform 2016; 90:1-11

17 Swary JH, Stratman EJ. Practice gaps in patient safety among dermatology residents and their teachers: a survey study of dermatology residents. JAMA Dermatol 2014;150(07):738-742

18 Colicchio TK, Cimino JJ. Clinicians' reasoning as reflected in electronic clinical note-entry and reading/retrieval: a systematic review and qualitative synthesis. J Am Med Inform Assoc 2019;26 (02):172-184 